\title{
Experiments on the Richtmyer-Meshkov instability: single-scale perturbations on a continuous interface
}

\author{
By M. BROUILLETTE† AND B.STURTEVANT \\ Graduate Aeronautical Laboratories, California Institute of Technology, Pasadena, \\ CA 91125 , USA
}

(Received 29 August 1991 and in revised form 19 May 1993)

We report on experiments to measure the shock-induced growth of sinusoidal perturbations on thick interfaces separating two gases of different densities. The results show that the growth rates are reduced as the interface thickness is increased. A model that accounts for the growth rate reduction caused by the presence of a finite density gradient on the interface is proposed and good agreement is obtained with the experimental results.

\section{Introduction}

When two fluids of different densities are impulsively accelerated into each other by a shock wave, if the interface separating them is not perfectly flat and parallel to the shock, a wide variety of fluid motions is generated. This class of problem is often referred to as the shock-excited Rayleigh-Taylor instability or as the RichtmyerMeshkov instability. The basic mechanism for the amplification of perturbations at the interface is baroclinic vorticity generation resulting from the misalignment of the pressure gradient of the shock and the density gradient across the interface. As the interface between the two fluids becomes more distorted, secondary instabilities, such as the Kelvin-Helmholtz shearing instability, develop and a region of turbulence and intense mixing results. The intensity of the turbulent motions can be further increased by processing the interface with additional pressure waves.

The instability resulting from the acceleration of one fluid into another one arises in the context of impulsively generated flows occurring both in man-made applications and in natural phenomena. For example, in the latter category, the RichtmyerMeshkov instability is used to explain: (i) the overturn of the outer portion of the collapsing cores of supernovas (Smarr et al. 1981) and (ii) the instability of rapidly collapsing gas bubbles in liquids (Cole 1948; Plesset 1954; Frost 1988). Examples of familiar technological applications affected by this shock-induced instability are (i) the interaction of reflected shocks with the contact discontinuity between driver and driven gases in shock tubes (Stalker \& Crane 1978), (ii) the interaction of pressure waves and flame fronts (Markstein 1957a), (iii) supersonic and hypersonic combustion (Marble, Hendricks \& Zukoski 1987; Waitz, Marble \& Zukoski 1991), (iv) laser-matter interactions (Grun et al. 1984; Emery, Gardner \& Boris 1982), and (v) laser implosion of deuterium-tritium fusion targets (Lindl \& Mead 1975; Afanas'ev et al. 1976). In most of these cases the instability tends to cause undesirably violent and uncontrollable

$\dagger$ Present address: Département de Génie Mécanique, Université de Sherbrooke, Sherbrooke, Québec, Canada J1K 2R1. 


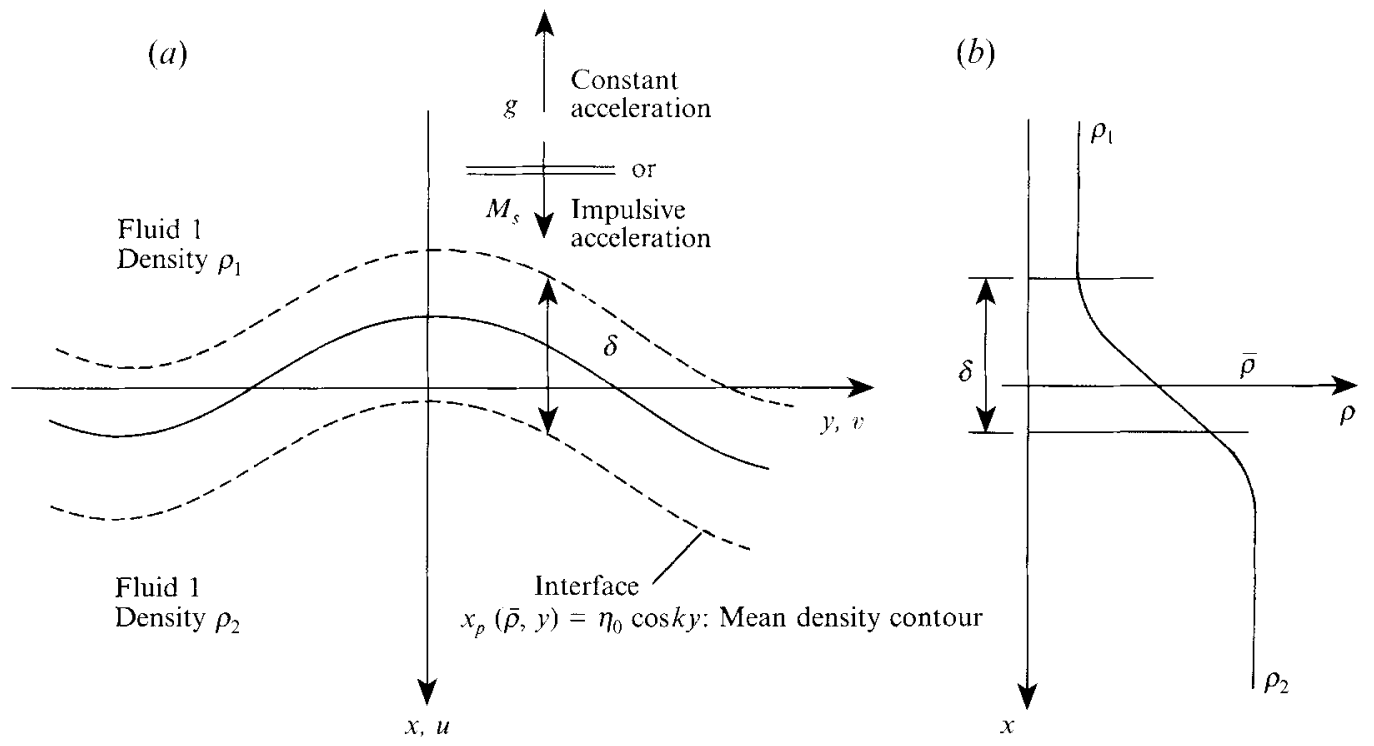

Figure 1. Initial configuration for the theoretical analysis of the acceleration of a continuous interface between two fluids of different densities. (a) Sinusoidal perturbation on interface. (b) Vertical density profile. A discontinuous interface would have a thickness $\delta \rightarrow 0$.

behaviour even when the interface has initially only small-scale perturbations, although one proposed method to control the effects of the instability is to reduce the density gradient at the interface to decrease the baroclinic generation of vorticity. Since the basic underlying physical processes are very much the same for all the cases mentioned above, significant understanding can be achieved by performing simple laboratory experiments at more benign conditions.

A vertical shock tube was built specially for the investigation of the shock-induced acceleration of an interface between two gases of different densities. Our preliminary experiments on the interaction of shock waves with plane interfaces of varying density gradient (Brouillette \& Sturtevant 1989) have shown that it is important for the method of measurement to distinguish between the phenomena under study and effects introduced by the experimental method, mainly at the walls of the shock tube. In the experiments reported here, we concentrate on the study of the time evolution of singlescale perturbations on diffuse interfaces, using schlieren photography and high-speed cinematography. The reduction in growth rate of perturbations by the decrease of the density gradient at the interface has been observed experimentally and has been confirmed by a simple analytical model.

\section{Theory}

The linear theory for the instability between two immiscible liquids of different densities under gravitational acceleration was first developed by Lord Rayleigh (1900). It was found that the instability occurs only if the light fluid accelerates into the heavy one, and that the initial growth of the perturbations is exponential in time. The linear theory has subsequently been modified to include effects of surface tension at the interface (Taylor 1950), viscosity (Bellman \& Pennington 1954), molecular diffusion (Duff, Harlow \& Hirt 1962) and slight nonlinearity (Kiang 1969). All these effects have been found to reduce the growth rate of the instability. Sharp (1984) reviews the 
experimental and computational aspects of the nonlinear development and turbulent mixing resulting from the gravitational instability of an interface between fluids of different densities.

The case of an interface under shock acceleration was considered by Markstein $(1957 \mathrm{~b})$, although the first rigorous treatment of the shock-excited instability was the theoretical analysis of Richtmyer (1960). Richtmyer considered the linearized response of a discontinuous interface between two perfect gases to an incident shock wave propagating normal to the plane of the undistorted interface (figure 1). The intensity of the motions at the interface depends on the strength of the incident wave. Richtmyer proposed that, for a weak shock, the velocities induced are relatively small, and the subsequent motion can be assumed to be incompressible. For that case, Richtmyer used the result of Taylor:

$$
\frac{\mathrm{d}^{2} \eta(t)}{\mathrm{d} t^{2}}=k g A \eta(t)
$$

for the growth of the amplitude $\eta$ of a discontinuous interface between incompressible fluids under gravitational acceleration $g$, where $k(=2 \pi / \lambda)$ is the horizontal wavenumber of the perturbation and $A$ is the Atwood ratio across the interface, $A \equiv\left(\rho_{2}-\rho_{1}\right) /\left(\rho_{1}+\rho_{2}\right)$. Richtmyer proposed replacing the constant acceleration $g$ by an impulsive one $g=[u] \delta_{D}(t)$, where $\delta_{D}$ is the Dirac delta function, $[u]$ is the change of interface velocity induced by the shock, in (1) and integrated once with respect to time to obtain the impulsive growth rate relation:

$$
\frac{\mathrm{d} \eta(t)}{\mathrm{d} t}=k[u] A \eta_{0}
$$

The analysis is valid as long as $\eta(t) \ll \lambda$, i.e. for $t \ll \lambda^{2} /\left(\eta_{0}[u]\right)$. From (2) it can be seen that the growth of the perturbations on an impulsively accelerated interface is linear in time, and that both light-heavy and heavy-light configurations are unstable. For the light-heavy configuration $\rho_{1}<\rho_{2}$, thus $A>0$ and the perturbation increases in amplitude from the start. For the heavy-light configuration $\rho_{1}>\rho_{2}$ and $A<0$; the perturbation initially decreases in amplitude before reversing its phase and growing unboundedly for this case.

Richtmyer solved the linearized problem numerically and found agreement within 5-10\% with the impulsive incompressible formula (2), provided $\eta_{0}^{\prime}$ and $A^{\prime}$, the postshock amplitude and Atwood ratio, respectively, are used instead of the pre-shock values, $\eta_{0}$ and $A$, to obtain the corrected expression:

$$
\frac{\mathrm{d} \eta(t)}{\mathrm{d} t}=k[u] A^{\prime} \eta_{0}^{\prime}
$$

where the primes denote the use of post-shock properties. Although shock refraction at an interface between two fluids of different densities is an inherently compressible process, (3) is usually considered an incompressible relation since the perturbation velocities are assumed small with respect to the local speed of sound.

Experiments with curved gaseous interfaces under shock acceleration by Meshkov (1969) verified the linear growth law of single-scale perturbations for both the light-heavy and the heavy-light case. Sturtevant (1988) pointed out that by using the correct post-shock parameters the growth rates measured by Meshkov can be made to agree more closely with the values predicted by the theory of Richtmyer, in which case the theory (3) still overpredicts the growth by a factor of about two. The general 
features of this formula have also been checked numerically (Meyer \& Blewett 1972) and experimentally in liquids (Benjamin, Trease \& Shaner 1984; Benjamin \& Fritz 1987).

The nonlinear development of the perturbations for the impulsive case is similar to that for constant acceleration (cf. Read 1984), with spikes of heavy fluid penetrating into the lighter one, leading ultimately to the appearance of a turbulent mixing zone at the interface. However, there is no experimental or theoretical evidence that the shock-induced turbulent thickening of the interface ever reaches an asymptotic stage of self-similar turbulent growth independent of initial conditions, as is observed in the constant gravity case (see Brouillette \& Sturtevant 1993 for details).

The effects of the density gradient on the accelerated interface between fluids of different densities were first considered theoretically in a rudimentary manner by LeLevier, Lasher \& Bjorklund (1955). The results have shown that the growth of the continuous interface under gravitational acceleration is reduced as its thickness is increased. In the limit of a thickness $\delta$ that is large compared to the wavelength $\delta \gg \lambda$, the growth rate is not totally suppressed but achieves a limiting value such that $\eta \approx \eta_{0} \exp \left[t(2 g A / \delta)^{\frac{1}{2}}\right]$.

Duff $e t$ al. (1962) have considered the problem of the thick interface under constant acceleration in more detail. The geometry of interest is also shown in figure 1. The starting point of this analysis is the linear eigenvalue equation for the perturbation velocity $u$, for a sinusoidally distorted arbitrary density profile under gravitational acceleration (Chandrasekhar 1961, p. 433):

with

$$
\begin{gathered}
\frac{\mathrm{d}}{\mathrm{d} x}\left(\rho \frac{\mathrm{d} u}{\mathrm{~d} x}\right)=u k^{2}\left(\rho-\frac{g}{\Omega^{2}} \frac{\mathrm{d} \rho}{\mathrm{d} x}\right), \\
\frac{\mathrm{d}^{2} \eta(t)}{\mathrm{d} t^{2}}=\Omega^{2} \eta(t),
\end{gathered}
$$

subject to $u \rightarrow 0$ as $x \rightarrow \pm \infty$. For a discontinuous interface, the eigenvalue $\Omega^{2}$ reduces to $\mathrm{kgA}$ and the result of Taylor, (1), is recovered. By analogy with that solution, Duff et al. proposed parametrizing the reduction in baroclinic torque due to the decrease of the density gradient at the interface by replacing the eigenvalue $\Omega^{2}$ with

$$
\Omega^{2}=\frac{k g A}{\psi},
$$

where $\psi$ is the so-called growth reduction factor which is a function of interface thickness and Atwood ratio; $\psi>1$ for the continuous interface and $\psi=1$ for the discontinuous interface. Substituting (5) into $(4 a)$, one then gets the new eigenvalue equation

$$
\frac{\mathrm{d}}{\mathrm{d} x}\left(\rho \frac{\mathrm{d} u}{\mathrm{~d} x}\right)=u k^{2}\left(\rho-\frac{\psi}{A k} \frac{\mathrm{d} \rho}{\mathrm{d} x}\right),
$$

where $\psi$ is now the eigenvalue to be determined. It is interesting to note here that this formulation for the eigenvalue proposed by Duff et al. is also independent of the nature of the acceleration.

The growth of perturbations at the interface is then obtained from the integration of $(4 b)$ using (5), i.e.

$$
\frac{\mathrm{d}^{2} \eta(t)}{\mathrm{d} t^{2}}=\frac{k g A}{\psi} \eta(t),
$$

where $\psi$ can be a function of time if the thickness of the interface increases, by 
molecular diffusion for example, while the perturbation on the interface grows in amplitude. Duff et al. obtained reasonable agreement between experiments and the model (7) for an interface between diffusing gases of different densities under gravitational acceleration.

The effects of a density gradient on the instability of an impulsively accelerated interface was first examined theoretically by Mikaelian (1985) who treated the continuous interface under impulsive acceleration by modelling it as a series of $M$ discrete discontinuous interfaces. The growth rate is obtained from the eigenvalues of a system of $M \times M$ equations. Although $M=5$ has been shown to model accurately a smooth continuous interface, this treatment is not amenable to a compact closedform expression. Saffman \& Meiron (1989) have calculated the kinetic energy generated by the impulsive acceleration of a incompressible continuously stratified interface.

To describe the growth of sinusoidal perturbation on a continuous interface under impulsive acceleration, we propose combining the model of Duff et al. for constant acceleration of thick interfaces with the impulsive acceleration formulation of Richtmyer. Thus, we replace the constant acceleration $g$ by an impulsive one $g=[u] \delta_{D}(t)$ in the growth rate relation (7). Since the thickening of the interface by molecular diffusion is much slower than the growth rate of the perturbation for shockaccelerated interfaces, the factor $\psi$ can be considered constant after shock refraction at the interface, and the equation is integrated directly to yield:

$$
\frac{\mathrm{d} \eta}{\mathrm{d} t}=\frac{k[u] A^{\prime}}{\psi} \eta_{0}^{\prime} .
$$

For the continuous interface we also propose to use post-shock properties for the Atwood ratio and initial amplitude, as specified by Richtmyer and Sturtevant for the discontinuous interface. From (8), it can be see that the growth rate of perturbations on continuous interfaces is still linear in time, but is reduced by a factor $\psi$ compared to that of the discontinuous interface. Because the gas densities and the interface thickness are modified as a result of the wave refraction at the interface, it is proposed that the Atwood ratio and interface thickness used to calculate $\psi$ be taken as the average of the pre- and post-shock values, i.e. $\psi=\psi(\bar{A}, \bar{\delta})$, with $\bar{A}=\frac{1}{2}\left(A+A^{\prime}\right)$ and $\bar{\delta}=\frac{1}{2}\left(\delta+\delta^{\prime}\right)$.

We determine the growth reduction factor $\psi$ by solving (6) numerically for the case of a diffusive profile between the two gases:

$$
\rho(x)=\bar{\rho}[1+\bar{A} \operatorname{erf}(x / \bar{\delta})]
$$

The characteristic thickness of the interface $\bar{\delta}$ can be taken as the maximum slope thickness of the density profile at a time $\tau$ after molecular diffusion began to take place between the two gases, such that, from $(9), \bar{\delta}=2(\pi D \tau)^{\frac{1}{2}}$, where $D$ is the molecular species diffusion coefficient. In the numerical scheme, a guess for $\psi$ is chosen for a given value of $\bar{A}$ and $\bar{\delta} / \lambda$ and the equation is integrated from $x \rightarrow-\infty$ to $x=0$ and from $x \rightarrow \infty$ to $x=0$. If the values for $u$ and $\mathrm{d} u / \mathrm{d} x$ from both integrations do not match at $x=0$, the guess for $\psi$ is adjusted until convergence is obtained.

Figure 2 shows a plot of $\psi$ as a function of thickness $v s$. wavelength ratio $\bar{\delta} / \lambda$ for the gas combinations considered in the present experiments. One can see that, for a given $\bar{\delta} / \lambda$, the growth reduction factor $\psi$ is higher for a lower Atwood ratio at the interface. In fact, the limit $\bar{A}=0$ corresponds to the analysis of LeLevier et al. (1955), i.e. $\psi=1+\pi(\bar{\delta} / \lambda)$. For a very thin interface, i.e. $\bar{\delta} / \lambda \approx 0, \psi$ reduces to 1 , and the discontinuous result of Taylor is recovered. In the limit that $\bar{A}=1$, then $\psi=1$ also. 


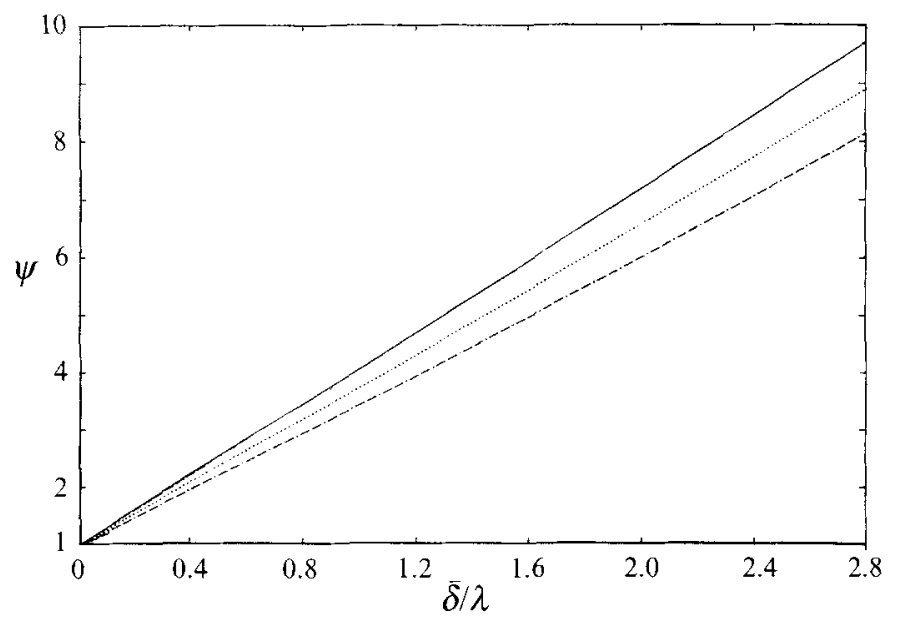

FIGURE 2. Growth reduction factor $\psi$ vs. thickness/wavelength ratio $\bar{\delta} / \lambda$ of interface: - air/air, $\bar{A}=0 ; \cdots$, air $/ \mathrm{R}-22, \bar{A}=0.50 ; \cdots-$, air $/ \mathrm{SF}_{6}, \bar{A}=0.67$.

\begin{tabular}{clc} 
Gas combination & \multicolumn{1}{c}{$\bar{C}$} & $C$ \\
Air/Air & 0 & $\pi$ \\
Air/R-22 & 0.50 & 2.83 \\
Air/Air & 0.67 & 2.59
\end{tabular}

TABLE 1. Approximation to the growth reduction factor: $\psi=1+C(\bar{\delta} / \lambda)$

If the wavelength of a perturbation on a continuous interface becomes small compared to its thickness, the $\psi v s . \bar{\delta} / \lambda$ curve becomes linear, and can be approximated by

$$
\psi \approx 1+C(\bar{\delta} / \lambda)
$$

with $C$ a function of the Atwood ratio. It is found that this is a good approximation for $\bar{\delta} / \lambda>1$. Then, for the case that $\bar{\delta} \gg \lambda$, the growth rate at the interface is not zero but reduces to

$$
\frac{\mathrm{d} \eta}{\mathrm{d} t}=\frac{2 \pi}{\bar{\delta}} \frac{A^{\prime}[u] \eta_{0}^{\prime}}{C}
$$

where the perturbation wavelength $\lambda$ has been replaced by the interface thickness $\bar{\delta}$ as the characteristic lengthscale. Table 1 lists the value of the constant $C$ for the gas combinations of interest, obtained from a straight line fit to the curves of figure 2 at large $\bar{\delta} / \lambda$.

As long as the perturbation stays in the linear regime $k \eta \ll 1$, it is proposed that, when the interface undergoes multiple impulsive accelerations caused by the successive arrival of many waves at the interface, the growth rate after $N+1$ impulsive accelerations be given by

$$
\left(\frac{\mathrm{d} \eta}{\mathrm{d} t}\right)_{N}=k \sum_{i=0}^{N} \frac{[u]_{i} A_{i}^{\prime} \eta_{i}^{\prime}}{\psi_{i}^{\prime}}
$$




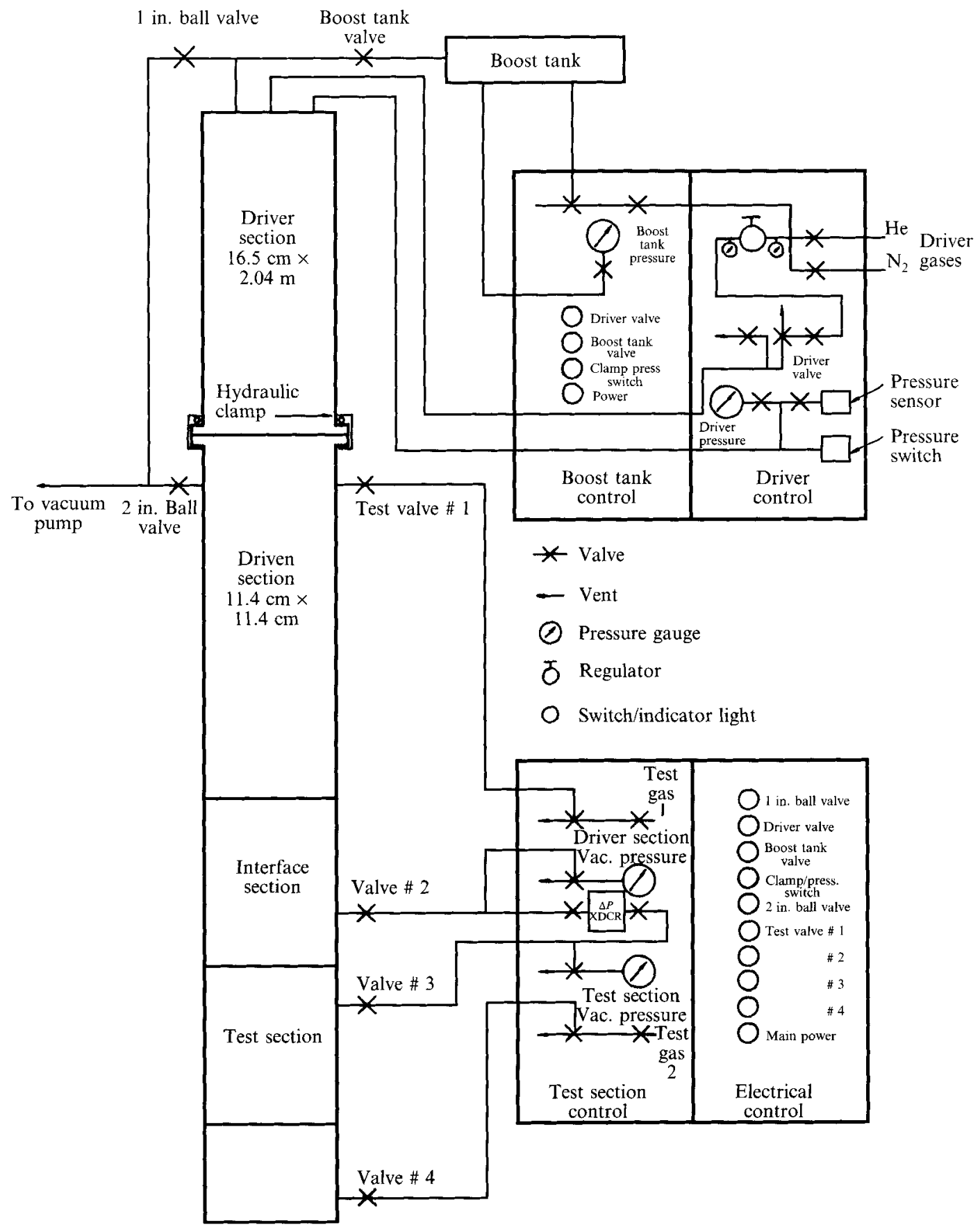

FIGURE 3. Schematic of GALCIT vertical shock tube.

where $[u]_{i}$ is the interface velocity change caused by the $i$ th wave; $A_{i}^{\prime}$ and $\eta_{i}^{\prime}$ are respectively the Atwood ratio across the interface and the amplitude of the perturbation just after refraction of wave $i$. The wave reduction factor $\psi_{i}$ is computed from $\overline{A_{i}}=\frac{1}{2}\left(A_{i}+A_{i}^{\prime}\right)$ and $\overline{\delta_{i}}=\frac{1}{2}\left(\delta_{i}+\delta_{i}^{\prime}\right)$. The wave numbering convention is such that the first wave refracted at the interface is wave 0 , the second is wave 1 , and so on. 


\begin{tabular}{lccc}
\multicolumn{1}{c}{ Property } & Air & $\mathrm{R}-22$ & $\mathrm{SF}_{6}$ \\
Molecular mass $\left(\mathrm{kg} \mathrm{kmol}^{-1}\right)$ & 29.04 & 86.48 & 146.07 \\
Density $\left(\mathrm{kg} \mathrm{m}^{-3}\right)$ & 1.18 & 3.54 & 5.97 \\
Atwood ratio with air & 0 & 0.50 & 0.67 \\
Specific heat ratio & 1.4 & 1.17 & 1.09 \\
Kinematic viscosity $\left(10^{-6} \mathrm{~m}^{2} \mathrm{~s}^{-1}\right)$ & 15.7 & 3.60 & 2.47 \\
Prandtl number & 0.71 & 0.73 & 0.90 \\
Diffusion coefficient in air $\left(\mathrm{cm}^{2} \mathrm{~s}^{-1}\right)$ & 0.204 & 0.104 & 0.097 \\
Index of refraction (Sodium D-line) & 1.000264 & 1.000669 & 1.000717
\end{tabular}

Table 2. Gas properties at $25^{\circ} \mathrm{C}, 1 \mathrm{~atm}$

\section{Experimental}

A vertical shock tube has been built specially for the study of the RichtmyerMeshkov instability. The tube is hung from only one point so it can easily be inverted or tilted at a small angle to the vertical. The test section, located at the bottom of the facility for these experiments, incorporates a system to produce an interface between two gases of different densities. A shock wave is launched from the top of the shock tube toward the interface below. In the experiments reported here the interface is initially located near the endwall of the tube and the tube is set precisely vertical, so the development of the instability between the two gases induced by an incident shock parallel to the interface, and its reverberations, are studied with schlieren photography.

Figure 3 shows a schematic diagram of the GALCIT vertical shock tube. It uses a conventional cold-gas driver section. The driver is equipped with a 'boost tank', which is filled with high-pressure driver gas and connected to the driver by a short, largediameter line and a high-capacity diaphragm solenoid valve. A trigger signal is used to control the rapid release of the high-pressure gas from the boost tank into the driver, already under pressure, to fire the shock tube. The driven section has a square inside cross-section of $11.4 \times 11.4 \mathrm{~cm}$ and is equipped with optical windows of the same width. More details about the facility can be obtained from Brouillette (1989).

To form a continuous interface, the test section is equipped with a system for withdrawing a thin $(1.2 \mathrm{~mm})$ horizontal metal plate initially separating the gases. With the light gas over the heavy one, the plate is withdrawn at a speed of $10 \mathrm{~cm} \mathrm{~s}^{-1}$, leaving a region of smooth density change between the two fluids. Atmospheric air is used above the interface and Freon-22 (R-22) (density $3.5 \mathrm{~kg} \mathrm{~m}^{-3}$ ) and sulphur hexafluoride $\left(\mathrm{SF}_{6}\right)$ (density $6.0 \mathrm{~kg} \mathrm{~m}^{-3}$ ) are used below the interface as test gases, although $\mathrm{CO}_{2}$ was also used in the preliminary stages of these experiments. Principal gas properties for air, $\mathrm{SF}_{6}$ and $\mathrm{R}-22$ are shown in table 2 .

In preparing the experiments, the test gas is introduced by pushing it into the test section while displacing the air out of it. After about 50 test section volumes of test gas have flowed, the gas purity is usually satisfactory. The actual mean composition of the test gas in the test section is obtained from a comparison of the experimental trajectories of the interface and waves with those calculated from one-dimensional gasdynamics theory. Good agreement is customarily obtained.

The sliding plate can be installed within the field of view of the flow visualization windows or at two locations $(32$ or $65 \mathrm{~cm}$ ) upstream of it. The location of the endwall of the shock tube with respect to the windows can also be moved, allowing us to change 


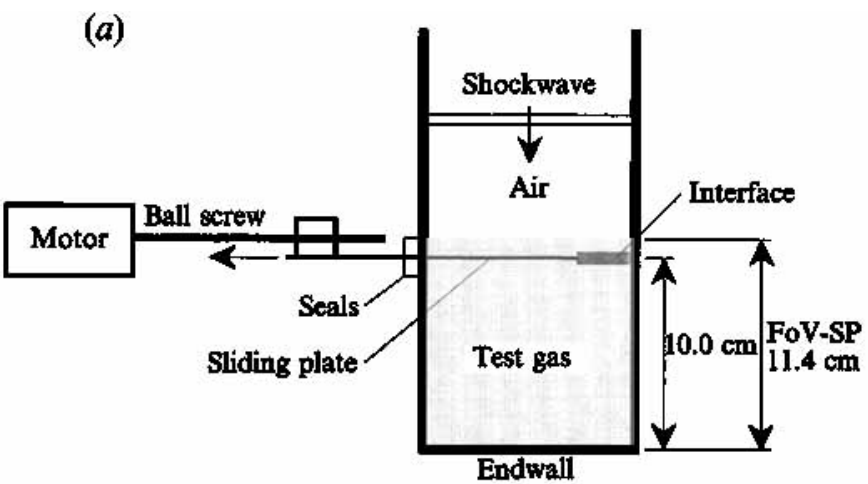

(b)

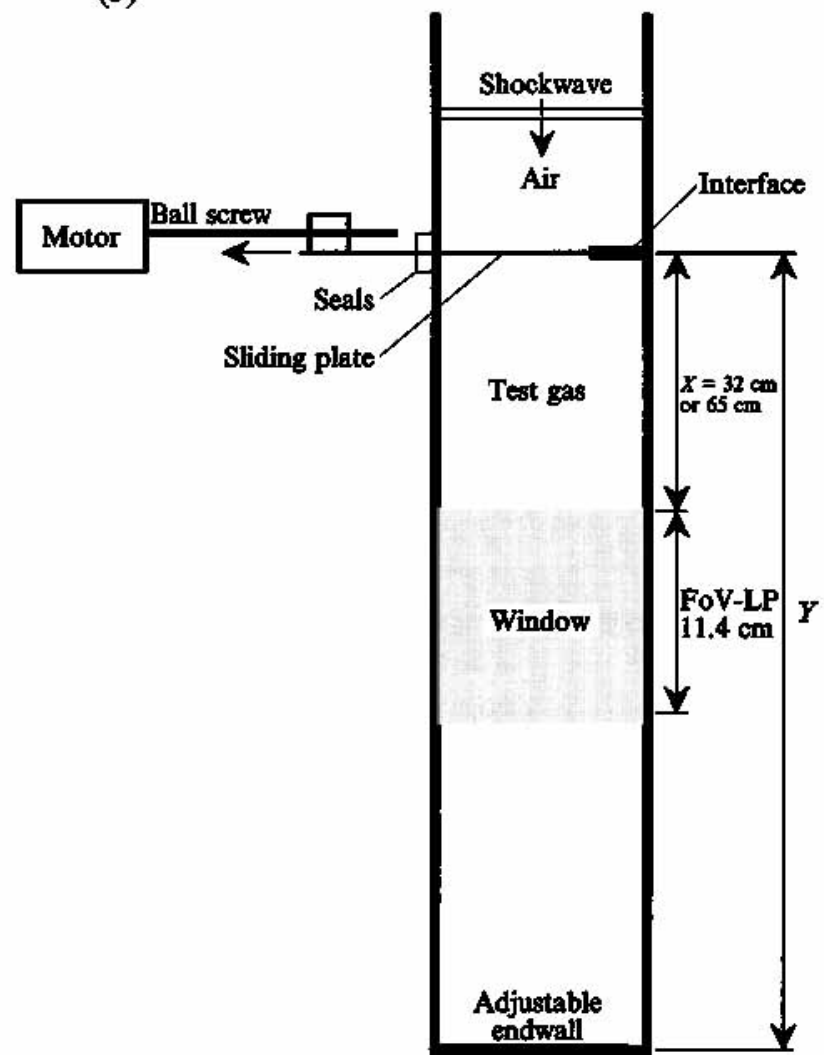

Figure 4. Experimental configurations. (a) Short-period experiments. The sliding plate mechanism is placed within the field of view of the windows and the endwall of the shock tube is positioned just $10 \mathrm{~cm}$ downstream of the sliding plate. This set-up is used to observe the evolution of the interface at early times after the interaction with the incident shock and also to study the effects of multiple impulsive accelerations caused by reverberations of the primary wave between the interface and the endwall of the shock tube. The field of view of the flow visualization system is indicated by 'FoV-SP'. (b) Long-period experiments. The distance $X$ between the sliding plate and the window can be set to $32 \mathrm{~cm}$ or $65 \mathrm{~cm}$ and the distance $Y$ between the sliding plate and the endwall is adjusted to observe the interaction of the first reshock with the interface just as the latter is about to leave the lower end of the window. The field of view of the flow visualization system is indicated by 'FoV-LP'. 


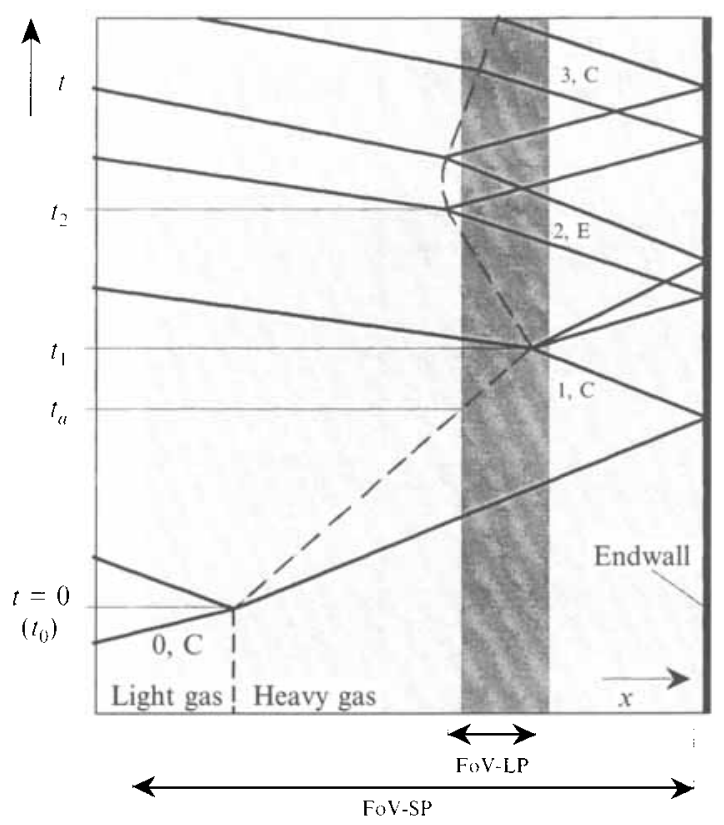

FiguRE 5. Generic wave diagram for the interaction of a moderate-strength shock wave with a light-heavy interface. The interface trajectory is indicated by a dashed line. The wave trajectories are indicated by a solid line. Waves successively interacting with the interface are numbered $0,1,2$, etc.; $\mathrm{C}$ indicates compression waves, $\mathrm{E}$ expansion waves. The field of view of the flow visualization system in the short-period configuration is indicated by 'FoV-SP' (light shading) and by 'FoV-LP' (heavy shading) for experiments in the long-period configuration, respectively.

the period of the waves reverberating between the interface and the endwall. Although a large number of combinations are possible, two configurations are used. In the first, 'short period' experiments are performed with the interface initially in the field of view of the windows, and the endwall of the shock tube is moved just $10 \mathrm{~cm}$ downstream of the interface (figure $4 a$ ). This set-up is used to observe the evolution of the interface at early times after the interaction with the incident shock and also to study the effects of multiple impulsive accelerations caused by reverberations of the primary wave between the interface and the endwall of the shock tube. Figure 5 shows a generic wave diagram for the interaction of a moderately weak shock wave with a light-heavy interface. At $t=0$, the incident shock is refracted at the interface and impulsively accelerates it towards the endwall. The shock transmitted in the heavy fluid then reflects from the end boundary (wave $1, \mathrm{C}$ ) and interacts with the moving interface at $t=t_{1}$, causing it to reverse its motion. At the same time expansion waves are reflected back into the heavy fluid and a shock is transmitted into the light gas. Waves trapped in the heavy gas then reverberate between the interface and the endwall and gradually bring the interface to rest. The field of view of the flow visualization system for the short-period experiments is indicated by 'FoV-SP'. The present convention numbers the first wave interacting with the interface 0 , the second 1 and so on, with compression waves denoted by $\mathrm{C}$ and expansion waves by $\mathrm{E}$.

In the other configuration, the 'long period' experiments, the interface is initially located either 32 or $65 \mathrm{~cm}$ upstream of the flow visualization windows. The end of the shock tube is adjusted so that the first reflection from the endwall interacts with the interface just as the latter is about to leave the bottom of the window. Thus, a 


\begin{tabular}{|c|c|c|c|c|c|c|}
\hline Test gas & $A_{11}$ & $M_{s}$ & $\begin{array}{c}\text { Sliding plate to } \\
\text { window distance } \\
X^{\prime}(\mathrm{cm})\end{array}$ & $\begin{array}{l}\text { Sliding plate to } \\
\text { endwall distance } \\
\qquad Y^{\prime}(\mathrm{cm})\end{array}$ & $\begin{array}{c}\text { Appearance } \\
\text { time } \\
l_{0}(\mathrm{~ms})\end{array}$ & $\begin{array}{c}\text { Reshock } \\
\text { time } \\
t_{1} \text { (ms) }\end{array}$ \\
\hline \multirow[t]{4}{*}{$\mathrm{R}-22$} & 0.50 & 1.12 & 32 & 102 & 5.94 & 8.19 \\
\hline & & 1.32 & 32 & 64 & 2.60 & 3.65 \\
\hline & & 1.48 & 32 & 53 & 1.78 & 2.43 \\
\hline & & 1.66 & 32 & 50 & 1.38 & 1.90 \\
\hline \multirow[t]{4}{*}{$S F_{6}$} & 0.67 & 1.12 & 32 & 84 & 6.87 & 8.71 \\
\hline & & 1.32 & 32 & 56 & 3.07 & 4.12 \\
\hline & & 1.48 & 32 & 49 & 2.11 & 2.81 \\
\hline & & 1.66 & 65 & 84 & 3.25 & 3.86 \\
\hline
\end{tabular}

TABIF 3. Experimental conditions -... long-period experiments

'snapshot'view of the interface a long time after it interacts with the incident shock, as well as of the effects of the first reshock, is obtained. Figure $4(b)$ shows a schematic of the long-period experimental arrangement and table 3 lists the relevant tube dimensions for various gas pairs and incident shock strengths. Table 3 also lists the time $t_{a}$ at which the interface first appears in the field of view of the windows after the start of the interaction and the time $t_{1}$ at which the interaction with the first reshock takes place in the long-period experiments. The field of view of the flow visualization system for the long-period experiments is indicated by 'FoV-LP' in the generic wave diagram of figure 5, which still applies in the long-period experiments.

The evolution of the interface under impulsive accelerations is observed using a schlieren optical system. The sensitivity of the schlieren system is adjusted so that the interface can easily be distinguished from the image of the shock wave-boundary layer interaction on the observing window. At the same time, the sensitivity is kept sufficient to record the random field of acoustic waves associated with the shock waves reverberating between the interface and the end of the tube. For examining the fine details of the interaction between the two gases, high-resolution spark schlieren photography is used. To measure the time evolution of the interface we use high-speed (35000-60000 frames per second (f.p.s.)) schlieren motion pictures which yield images of lower resolution.

To perform a run, the sliding plate is fully inserted into the test section, and the test gas is introduced below the interface. A microprocessor-driven control system is used to provide the sequence for retracting the plate, firing the shock wave and triggering the flow visualization equipment.

\section{Results}

\subsection{Initial perturbation on the continuous interface}

Perturbations on the interface can be introduced by taking advantage of the fact that, because of the no-slip condition on the surface of the plate, the plate drags along a certain amount of fluid as it retracts. A volume of fluid then accumulates at the sidewall and, just as the plate is fully retracted, propagates out along the interface as a dispersive gravity wave, leaving behind a quasi-sinusoidal perturbation on a thick diffusive interface.

Figure 6(a) shows a schlieren picture of the interface between air and $\mathrm{SF}_{6}$ just after the plate is retracted from the left. The interface is the dark region near the top of the 
(a)

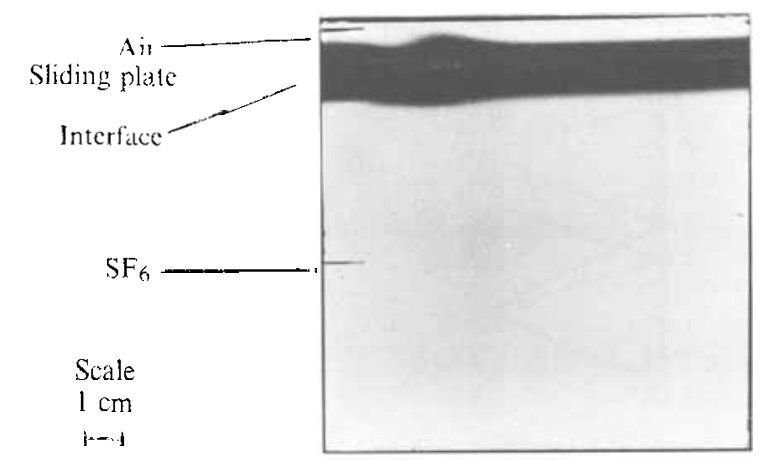

(b)

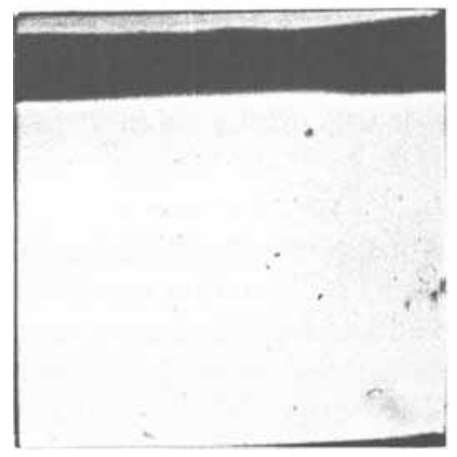

(c)

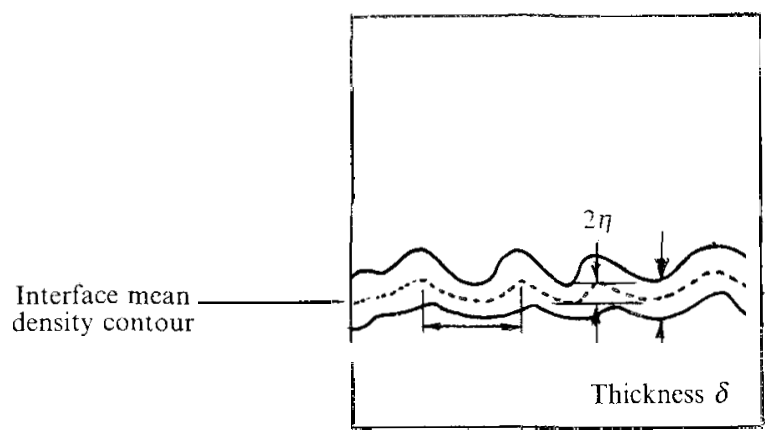

Figure 6. Initial conditions at perturbed continuous interface between air and $\mathrm{SF}_{6 .}$. (a) Interface just after plate retraction from the left. A volume of interface fluid was entrained by the retraction of the plate and has accumulated as a bulge against the left sidewall. (b) Initial perturbation used in the experiments. At a time $0.9 \mathrm{~s}$ after plate retraction, the bulge on the interface has just propagated to the right sidewall as a dispersive gravity wave leaving a perturbation on the interface. (c) Sketch of interface of figure $10(c)$. The visual thickness of the interface is denoted by $\delta$ and the average amplitude of the interface mean density contour is denoted by $\%$.

photograph. The accumulation of fluid pumped by the plate can be seen as a bulge on the left portion of the interface. According to our results on the shock-induced instability of plane continuous interfaces (Brouillette \& Sturtevant 1993), this bulge is the only perturbation introduced on the interface by the retraction of the sliding plate before any shock interaction, since any mixing at the interface is damped out quite rapidly by the stable density stratification before the arrival of the first shock wave. Figure $6(b)$ shows a schlieren picture of the same interface taken 0.9 s after full plate retraction, at the instant when the gravity wave has propagated to the right side of the 
test section, leaving a series of crests and troughs on the interface. The nature of the perturbation on the interface is difficult to describe at this point. Figure $10(c)$ shows the interface of figure $6(b)$ after multiple impulsive accelerations in the short-period experimental configuration. The quasi-sinusoidal nature of the perturbation can clearly be seen here. Because this perturbation is produced by the propagation of a dispersive gravity wave from the left side of the test section to the right, some asymmetry inevitably develops on the interface. This explains why the development of the shocked interface is also asymmetrical.

Throughout these experiments, measurements are taken from enlargements of the original high-speed motion picture negatives, with measurements for a given test condition obtained from a single experimental run. Figure $6(c)$ shows a schematic of the interface of figure $10(c)$. The thickness of the interface $\delta$ is measured as the vertical extent of dark region in the photographs (figure $6 c$ ). The interface mean density contour is taken as the loci of interface midpoints (i.e. $\frac{1}{2} \delta$ ). The amplitude $\eta$ of the perturbation is then measured as the average amplitude of this mean density contour. These schlieren measurements of interface thickness were verified against concentration probe measurements (Brouillette 1989). Recently, Bonazza (1992) performed X-ray densitometry measurements of air-xenon interfaces using the same experimental facility and has confirmed the validity of the other two measurement techniques. The concentration probe and X-ray measurements have also shown that the experimental density profile is very close to the purely diffusive profile (9), with the measured thickness close to the maximum slope thickness. In making these measurements, it is important to distinguish between the interface in the bulk of the fluid and the image of the distorted interface adjacent to the observing windows. A thorough discussion of this distinction is presented by Brouillette \& Sturtevant (1989) and Brouillette (1989).

To ensure that the perturbation produced by the retraction of the sliding plate is two-dimensional, experiments have also been performed with the orientation of the sliding plate mechanism rotated $90^{\circ}$ with respect to the test section. For this case, no single-scale perturbation could be detected in the field of view before and after multiple impulsive accelerations, and we conclude that the quasi-sinusoidal perturbation is indeed two-dimensional.

The experiments are performed in a manner such that the incident shock wave interacts with the interface perturbation described above (e.g. figure $6 b$ ). The effect of the reverberations of the incident wave on the development at the interface is also studied. We examine in detail the growth of the perturbations on the interface after the refraction of the incident shock wave, its first reflection and multiple wave reverberations.

\subsection{Growth after incident shock}

The following photographs are obtained from a 35000 f.p.s. motion picture of a shortperiod experiment for the air $/ \mathrm{SF}_{6}$ interface accelerated by a $M_{s}=1.32$ shock wave, where $M_{s}$ is the Mach number of the wave: figure $6(b)$ is taken before the arrival of the shock and shows the nature of the initial perturbation on the interface; figure $7(a)$, taken a time $t=0.7 \mathrm{~ms}$ after the arrival of the incident wave, shows the interface shortly before interacting with the first reshock, seen below the interface. The interaction with the incident shock has compressed the interface, making the perturbation more visible, but not much growth is detected. The pair of thin lines near the top of the windows mark the location of the sliding plate, now fully retracted.

Figures $7(b)$ and $7(c)$ show pictures obtained from a 35000 f.p.s. motion picture of the same experiment, performed in the long-period configuration. They show the interface at $t=3.6$ and $4.0 \mathrm{~ms}$, and it is seen that the perturbation has grown only 


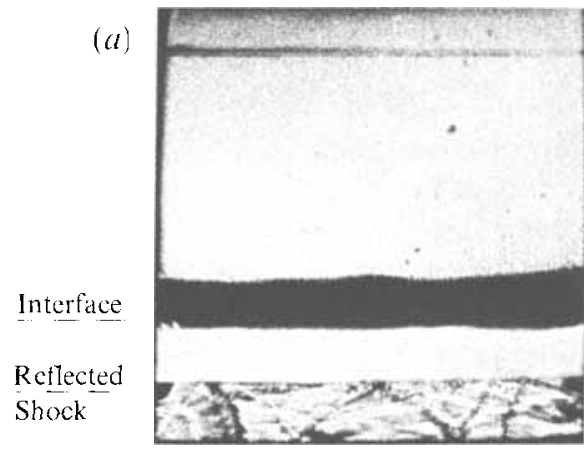

(b)

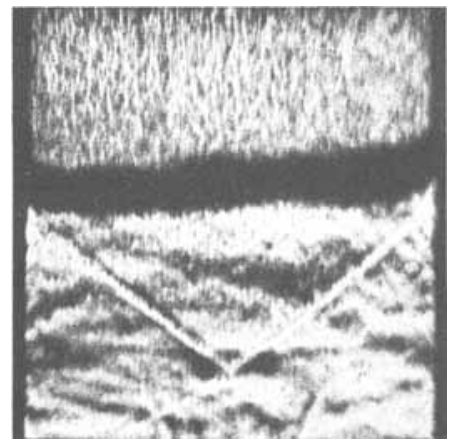

(c)

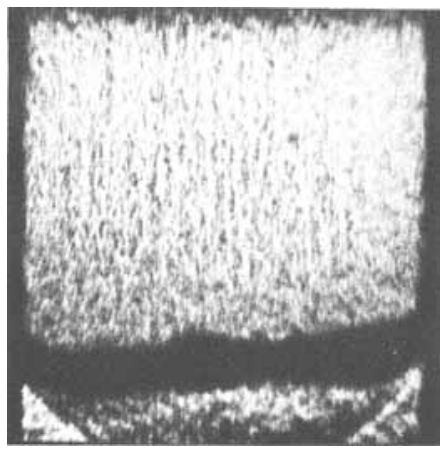

FIGURE 7. Single-scale Richtmyer-Meshkov instability at a continuous interface, growth after incident shock, short-period and long-period experiments. Air $/ \mathrm{SF}_{6}, M_{s}=1.32$. (a) $t=0.7 \mathrm{~ms}$, (b) $t=3.3 \mathrm{~ms}$, (c) $t=4.0 \mathrm{~ms}$.

slightly from the beginning of the interaction (i.e. figures $6 b$ and $7 a$ ). The pair of oblique waves seen below the interface originate from cylindrical waves that were generated by the arrival of the shock at the interface. Although these waves are weak. they can be seen clearly because of the high index of refraction of $\mathrm{SF}_{6}$. The shadow of the $\mathrm{SF}_{6}$-rich boundary layer above the interface can be seen on the window as a longitudinally streaky structure, typical of turbulent boundary layers (Kline 1967). The growth rate of the perturbation is small because its initial amplitude is small and the interface is thick.

\subsection{Growth after reflected shock}

More easily measurable growth rates are observed after the interface interacts with the reverberations of the primary wave.

Figure 8 shows pictures, obtained from the same experimental run as in figure $7(b, c)$, of the interface after interacting with the first reflected shock. Figures $8(a)$ shows the interface shortly after the arrival of the reflected shock, seen above the interface. The reshock is curved upwards because the speed of sound in the $\mathrm{SF}_{6}$-rich boundary layer above the interface is smaller than in the air in the bulk of the fluid above the interface. The interface has been compressed by the reshock and the quasi-periodic nature of the perturbation can easily be distinguished at this point. After that, the interface amplitude increases steadily, as seen in the subsequent frames. In figure $8(b)$, one can also notice the presence on the sidewalls of a bulge on the interface, called a 'loop' by Andronov et al. (1976). It is caused by the interaction of the reflected shock with the distorted interface within the boundary layer adjacent to the shock tube wall and is a 


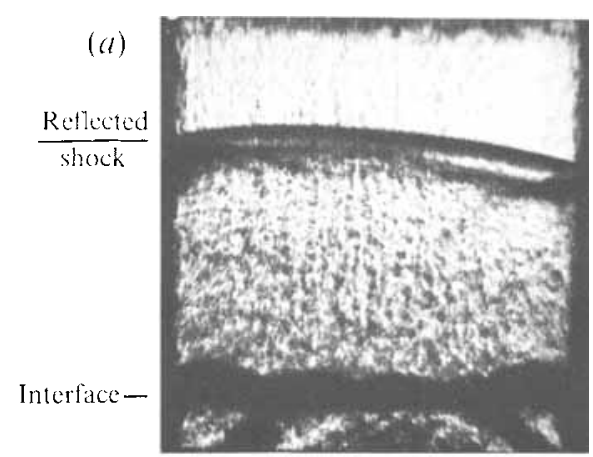

(c)
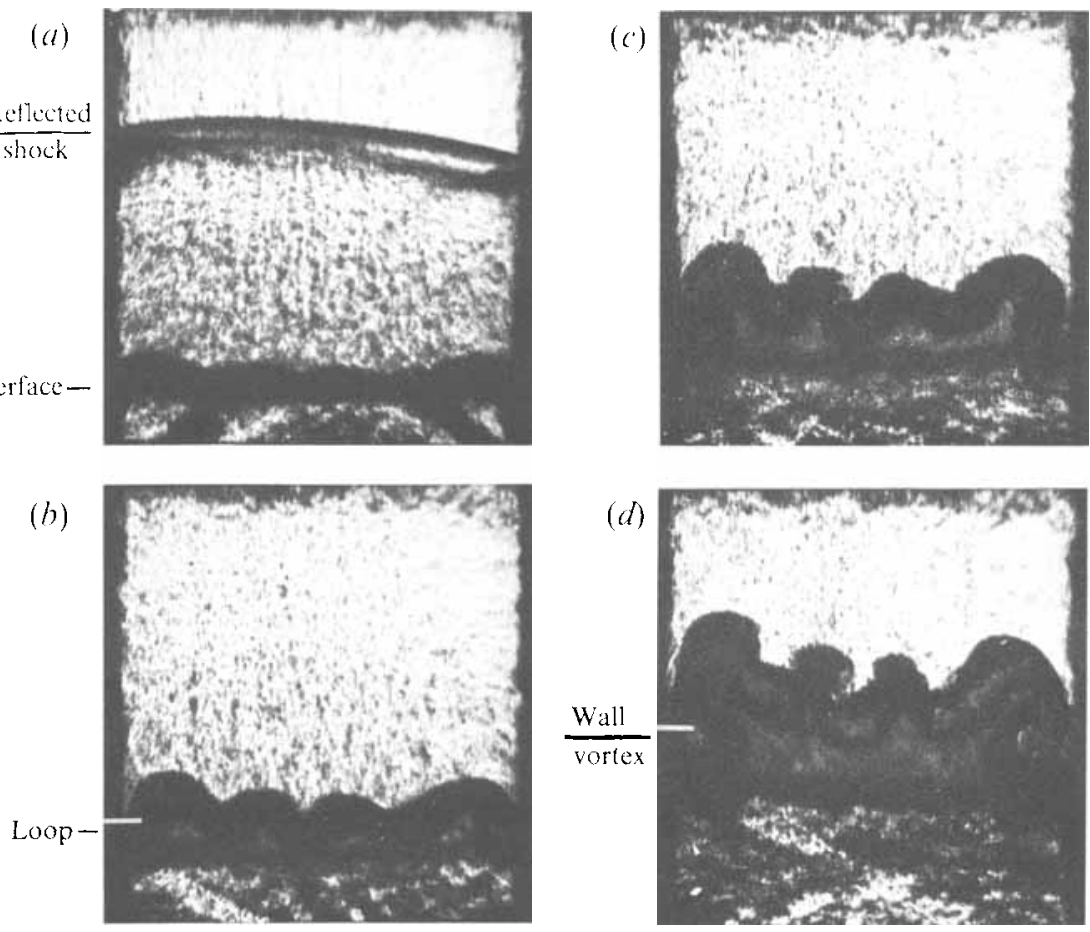

FIGURE 8. Single-scale Richtmyer-Meshkov instability at a continuous interface, growth after first reshock. Air $/ \mathrm{SF}_{f}, M_{s}=1.32$. (a) $t=4.3 \mathrm{~ms},(b) t=4.5 \mathrm{~ms},(c) t=4.8 \mathrm{~ms},(d) t=5.2 \mathrm{~ms}$.

form of shock wave-boundary layer interaction (cf. Brouillette 1989). An intensified Richtmyer-Meshkov instability inside the boundary layer leads to the formation of a two-dimensional wall vortex, clearly seen in figure $8(d)$ and also appearing as the lighter grey region on the window below the interface in figures $8(b), 8\left(c^{\prime}\right)$ and $8(d)$. The influence of the wall vortical structure gradually becomes important and leads to the overall deformation of the interface. At this stage the development of the perturbation on the interface is probably dominated in part by the wall vortex. A non-uniform acoustic field below the interface can also be noticed in the last three photographs. These weak waves, seen reverberating between the sidewalls, originate from the scattering of the incident and reflected shocks at the distorted interface and boundary layer. Aspects of these experiments related to shock wave-boundary layer interaction outside and within the interface are discussed in detail in Brouillette (1989).

Figure 9 is a plot of the evolution of the absolute value of the amplitude of the interface perturbation for the same experiment. The arrival of the reshock is indicated on the plot as the vertical line labelled 1, C (for compression wave). As observed in the pictures, the growth before the reshock is very small. However, after the reshock, a phase reversal of the perturbation, i.e. negative amplitude, is detected from the pictures at around $t=4.2 \mathrm{~ms}$, as indicated on the figure by the vertical arrow: the amplitude of the perturbation increases rapidly thereafter at a rate of $6.6 \pm 0.9 \mathrm{~m} \mathrm{~s}^{-1}$, as obtained from a least-squares line fit of the data. At about $t=5.0 \mathrm{~ms}$ the grow th appears to slow down to a stop. This slowdown can be caused by the evolution of the perturbation into the slower nonlinear regime or by the increased influence of the wall vortices.

Table 4 lists the measured growth rate after the reflected shock $(\mathrm{d} \eta / \mathrm{d} t)_{1}$, as obtained from a straight-line least-squares fit of the data, for both interface combinations and 


$\begin{array}{ccccc}\text { Test gals } & A_{1} & M & \begin{array}{c}(\mathrm{d} y / \mathrm{d} z)_{1} \\ (\mathrm{~ms})^{\prime}\end{array} & \begin{array}{c}1 /(\mathrm{mm}) \\ \text { calculated }\end{array} \\ \mathrm{R}-22 & 0.50 & 1.12 & 5.5 & 0.21 \\ & & 1.32 & 7.1 & 0.13 \\ & & 1.48 & 12.5 & 0.16 \\ \mathrm{SF}_{6} & 0.67 & 1.12 & 3.6 & 0.057 \\ & & 1.32 & 6.6 & 0.046 \\ & & 1.48 & 7.5 & 0.052\end{array}$

TABLF 4. Single-scale growth - long-period configuration

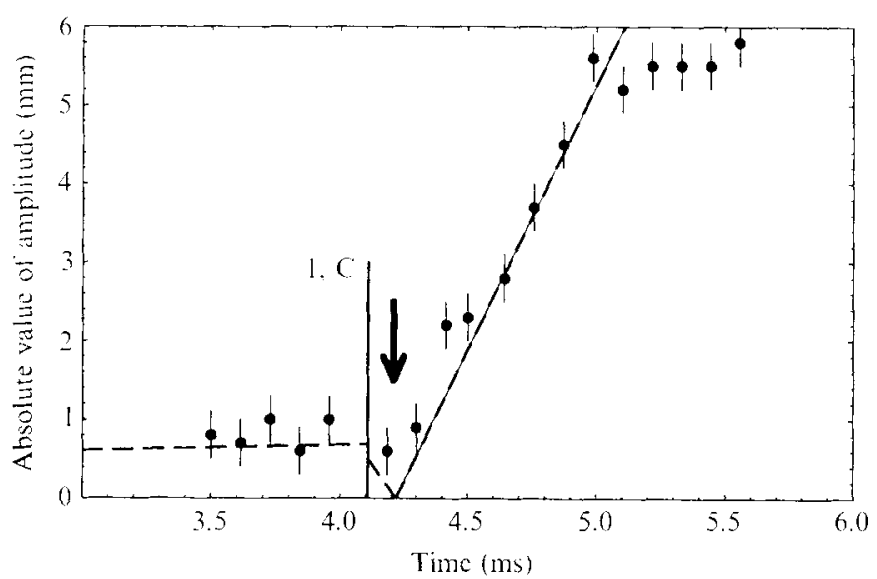

Figurf 9. Time evolution of the absolute value of the amplitude of a single-scale perturbation on a continuous interface. Air $/ \mathrm{SF}_{6}, M_{*}=1.32$, long-period experiment. - Least-squares line fit to the reshock data; - . calculated from (12) with $\eta_{0}$ chosen to match reshock grow th rate of experimental data. The observed time of phase reversal of the perturbation $t_{n-0}$ is indicated by the vertical arrow and corresponds closely to that calculated from (13).

various incident shock strengths in the long-period experimental configuration; the typical uncertainty in the data is $15-20 \%$.

\subsection{Multiple wave reverberations}

The evolution of interface perturbations under repeated impulsive accelerations is examined with experiments in the short-period configuration.

Figure 10 shows pictures from the same experimental run as in figures $6(b)$ and $7(a)$. In figure $10(a)(t=1.0 \mathrm{~ms})$, the interface is shown just after the interaction with the first reshock, shown in figure $7(a)$ just below the interface. The interface has been compressed by the refraction of this wave at the interface and the expansion waves reflected into $\mathrm{SF}_{6}$ can be noticed as the dark wavy region that mirrors the shape of the interface (figure $10 a$ ). Subsequently, the waves reverberating between the endwall and the interface die down and bring the interface to rest. The fine graining of the trapped wave pattern can be observed from figure $10(b-d)$. The increase in amplitude of the perturbation on the interface can also be distinguished. The nonlinear development of the perturbation is observed in figure $10(d)$ where round spikes of $\mathrm{SF}_{6}$ are seen penetrating into air. The wall vortical structures are also apparent in these pictures.

Figure 11 shows a plot for the time evolution of the measured absolute value of the amplitude of the perturbations for that experiment. The arrival of each reverberation is indicated by the vertical lines, where $\mathrm{C}$ and $\mathrm{E}$ denote compression and expansion 


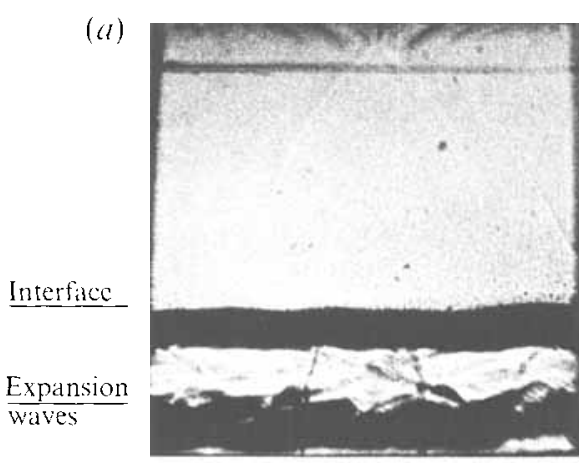

(b)

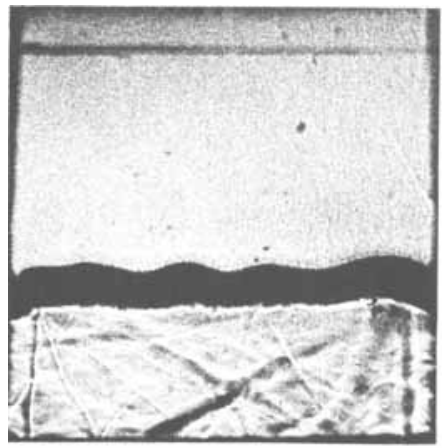

(c)

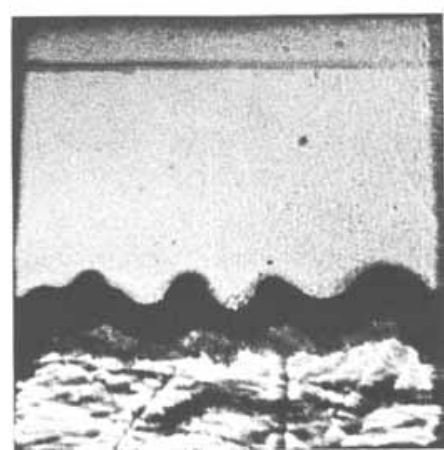

$(d)$

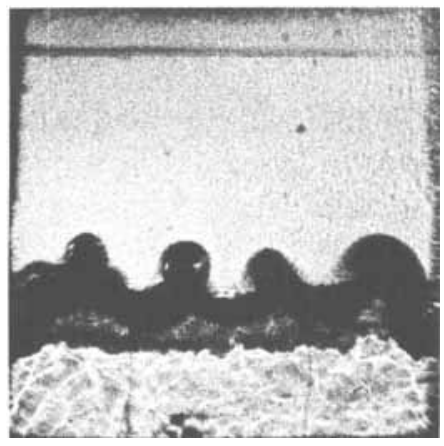

FIgURE 10. Single-scale Richtmyer-Meshkov instability at a continuous interface, growth after multiple reverberations, short-period experiment. $\mathrm{Air} / \mathrm{SF}_{f}, M_{s}=1.32$. (a) $t=1.0 \mathrm{~ms},(b) t=1.3 \mathrm{~ms}$, (c) $t=1.5 \mathrm{~ms},(d) t=1.7 \mathrm{~ms}$.

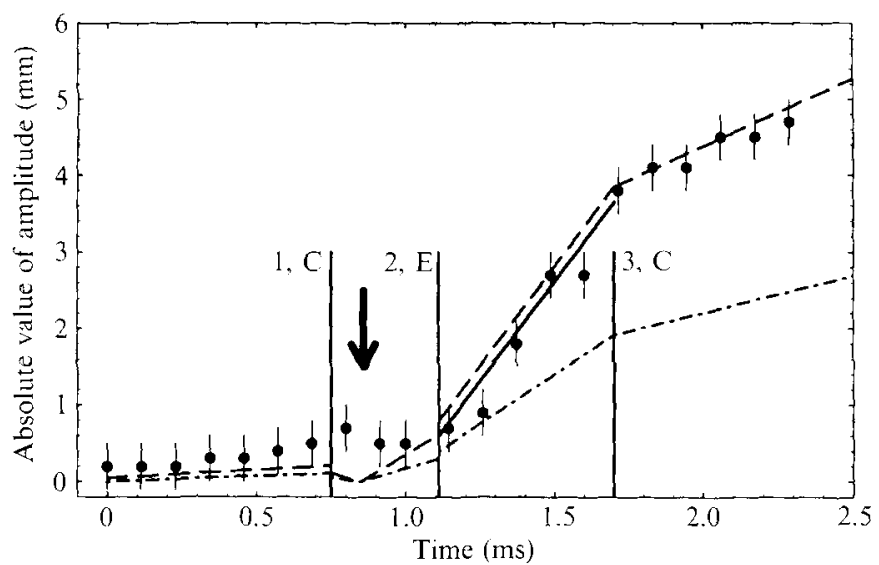

FIGURF. 11. Time evolution of the absolute value of the amplitude of a single-scale perturbation on a continuous interface. Air $/ \mathrm{SF}_{6}, M_{*}=1.32$, short-period experiment. - - Least-squares line fit to the data after wave $2 ;-\ldots$, calculated from (12) with $\eta_{0}$ chosen to match slope of experimental data; -.--, calculated from (12) using the average value of $\eta_{0}$ obtained from the long-period data. The observed time of phase reversal of the perturbation $t_{n-()}$ is indicated by the vertical arrow and corresponds closely to that calculated from (13).

waves, respectively. The initial growth is small, and the first reshock causes a phase reversal of the perturbation at $t=0.8 \mathrm{~ms}$, as indicated on the figure by the vertical arrow. After the interaction with the expansion waves, the amplitude increases rapidly at $5.5 \pm 1.2 \mathrm{~m} \mathrm{~s}^{-1}$, as obtained from a least-squares line fit of the observed data. At 


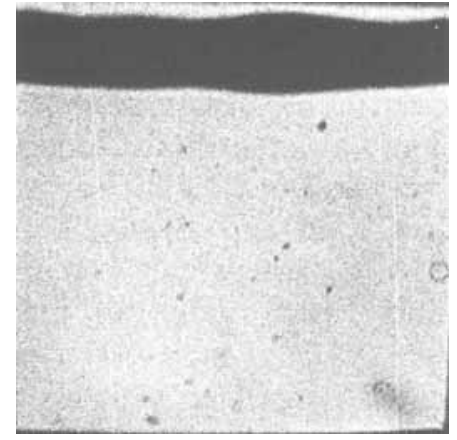

FIGURt 12. Initial perturbation on continuous interface between air and $\mathrm{R}-22$. A comparison with the initial perturbation on the air $/ \mathrm{SF}_{\text {interface (figure } 6}$ ) confirms that the initial perturbation on the air/R-22 interface is larger, as calculated using the theory (12) and the growth rate measurements.

$$
\eta_{11}(\mathrm{~mm})
$$

$\begin{array}{ccccc}\text { Test gas } & A_{0} & M_{,} & \text {Short period } & \text { Long period } \\ \text { R-22 } & 0.50 & 1.12 & 0.26 & 0.21 \\ & & 1.32 & 0.18 & 0.13 \\ & & 1.48 & 0.13 & 0.16 \\ & & 1.66 & 0.08 & \mathrm{~N} / \mathrm{A} \\ \text { SF }_{6} & 0.67 & \text { average } & 0.16+0.08 & 0.17 \pm 0.04 \\ & & 1.12 & 0.054 & 0.057 \\ & & 1.32 & 0.094 & 0.046 \\ & & 1.48 & 0.079 & 0.052 \\ & & 1.66 & 0.078 & \mathrm{~N} / \mathrm{A} \\ & & \text { average } & 0.076 \pm 0.016 & 0.052 \pm 0.006\end{array}$

TABLF 6. Single-scale growth - calculated initia amplitude of perturbation for short-period and long-period configurations

from both short-period and long-period experiments. The agreement between the two sets of data is reasonable. It is important to note that the discrepancy between the initial amplitude calculated from short- and long-period experiments is comparable to that observed between Richtmyer's theory (1960), on which the present model is based, and the relevant experimental results (Meshkov 1969; Sturtevant 1988).

While the discrepancy between the theory of Richtmyer and the relevant experiments is usually attributed to the effects of the membranes used to form the experimental discontinuous interfaces, the discrepancy between the theoretical short- and longperiod results in the present experiments can be attributed to at least three effects. First, the linear theory has been used into a regime in which the amplitude of the perturbation no longer satisfies the criterion $\eta \ll 1 / k$, particularly at the latter times in the short-period data. More specifically, the definition of interface mean density contour of $\$ 4.1$ loses its validity when spikes start to develop and the interface loses its symmetrical sinusoidal shape. Second, the calculations were simplified by assuming that the perturbation on the interface comprised only a single sinusoidal horizontal mode rather than a number of superposed modes in the horizontal and possibly the 


\begin{tabular}{|c|c|c|c|c|c|}
\hline \multirow[b]{2}{*}{ Test gats } & \multicolumn{5}{|c|}{$(\mathrm{d} m / \mathrm{d} \ell)_{2}\left(\mathrm{~ms}^{\prime}\right)$} \\
\hline & $A_{0}$ & $M$ & measured & calculated & $\begin{array}{l}1 / 10(\mathrm{~mm}) \\
\text { calculiated }\end{array}$ \\
\hline $\mathrm{R}-22$ & 0.50 & 1.12 & 0.7 & 0.5 & 0.26 \\
\hline & & 1.32 & 2.1 & 2.1 & 0.18 \\
\hline & & 1.48 & 3.0 & 3.8 & 0.13 \\
\hline & & 1.66 & 2.1 & 4.5 & 0.08 \\
\hline$S F_{k}$ & 0.67 & 1.12 & 1.1 & 1.1 & 0.054 \\
\hline & & 1.32 & 5.5 & 3.0 & 0.094 \\
\hline & & 1.48 & 11.7 & 7.7 & 0.079 \\
\hline & & 1.66 & 8.9 & 5.9 & 0.078 \\
\hline
\end{tabular}

TABI.F. 5. Single-scalc growth - short-period configuration

around $t=1.8 \mathrm{~ms}$ the growth slows down, possibly because of the interaction with the second reshock (wave 3), the transition into the nonlinear regime or the influence of the wall vortices.

Table 5 lists the value of the growth rate after the expansion wave $2,(\mathrm{~d} \eta / \mathrm{d} t)_{2}$ 'measured', as obtained from the straight-line fit of the observed data for all gas combinations and incident shock strengths in the short-period configuration; the typical uncertainty in the data is around $20 \%$.

\section{Discussion}

Because the initial amplitude of the single-scale perturbation produced on the continuous interfaces cannot be determined accurately, the proposed model (11) is validated in an indirect manner, from the two sets of experiments performed with each gas combination. The model is verified as follows:

Supposing that (12) is valid, the growth rates after reshock $(\mathrm{d} \eta / \mathrm{d} t)_{1}$ measured from long-period experiments and the growth rates after two reverberations $(\mathrm{d} \eta / \mathrm{d} t)_{2}$ measured from short-period experiments are each used to calculate the initial amplitude of the perturbation $\eta_{0}$ for the respective sets of experiments. The agreement between these two sets of data for the initial amplitude should then be considered an acceptable test for the theory.

Table 4 list the initial perturbation amplitude obtained from the reshock growth rates in the long-period experiments. From these results, the initial amplitude of the single-scale perturbation on the air $/ \mathrm{SF}_{6}$ interface is found to be $0.052 \pm 0.006 \mathrm{~mm}$. For the air $/ \mathrm{R}-22$ interface, $\eta_{0}=0.17 \pm 0.04 \mathrm{~mm}$. The relatively small scatter in the data can be considered as a first check of the formula. It is important to note that the proposed model (12) predicts reshock growth rates about eight times slower than those calculated from Richtmyer's formula for discontinuous interfaces, (3).

Table 5 lists the initial amplitudes calculated from the growth rate after the second reverberation $(\mathrm{d} \eta / \mathrm{d} t)_{2}$ in the short-period experiments. In this case, since the time duration of the interaction of the expansion waves with the interface is small, the impulsive formula (12) is used rather than integrating (7). From this set of data, the initial amplitude is found to be $\eta_{0}=0.076 \pm 0.016 \mathrm{~mm}$ for $\mathrm{SF}_{6}$ and $\eta_{0}=0.16 \pm 0.08 \mathrm{~mm}$ for R-22. The scatter for this series of experiments is larger than for long-period results, possibly because perturbation amplitude data have been used into the nonlinear regime.

These results are summarized in table 6 which lists the calculated initial amplitude 
same conditions on a discontinuous interface, $t_{\eta=0}-t_{1}=0.04 \mathrm{~ms}$. For most of the present experiments, however, the uncertainty in the observation of the time of phase reversal is large since this phenomenon occurs while refracted waves are still in the proximity of the interface, disturbing the field of view. When phase reversal can be located with accuracy on the motion pictures, such as for the results discussed in this paragraph, there is good agreement with the value predicted by (13), further confirming the validity of (12).

\section{Conclusions}

The experiments reported in this paper have confirmed that a significant reduction in the growth rate of single-scale perturbations on impulsively accelerated interfaces can be achieved by decreasing the density gradient initially present at the interface. For the interfaces under consideration, where $\delta / \lambda \approx 0.8$, the arrival of each wave induces a perturbation growth rate about three times smaller than predicted by the linear theory for the discontinuous interface. The growth rate measurements were compared to a model that accounts for the slower growth, and the agreement between the analytical and experimental results for the continuous interface is found to be comparable with that between the theory and experiments for the discontinuous interface. According to present model, the shock-excited growth of an interface after one impulsive acceleration can be reduced tenfold if the discontinuous interface is replaced by a continuous one for which $\delta / \lambda \approx 3$. In the limit $\delta / \lambda \rightarrow \infty$, the growth rate becomes independent of the wavelength of the perturbation, and the dominant lengthscale becomes the thickness of the interface.

This work was supported by the Lawrence Livermore Laboratory, Subcontract Number 7961805 under DOE W-7405-ENG-48.

\section{REFERENCES}

Afanas'ev, Yu. F., Basov, N. G., Gamalit, E. G., Krokhin, O. N. \& Rozanov, V. B. 1976 Symmetry and stability of laser-drive compression thermonuclear targets. JETP Lett. 23, $566-569$.

Andronov, V. A., Bakhrakh, S. M., Meshkov, E. E., Mokhov, V. N., Nikiforov, V. V., Pevnitski, A. V. \& Tolshmyakov, A. I. 1976 Turbulent mixing at contact surface accelerated by shock waves. Sov. Phys. JETP 44, 424-427.

Bellman, R. \& Pennington, R. H. 1954 Effects of Surface Tension and Viscosity on Taylor Instability. Quart. Appl. Math. 12, 151-162.

Benjamin, R. F. \& Fritz, J. N. 1987 Shock loading a rippled interface between liquids of different densities. Phys. Fluids 30, 331-336.

Benjamin, R. F., Trease, H. E. \& Shaner, J.W. 1984 Coherent density gradients in water compressed by a modulated shock wave. Phys. Fluids 27, 2390-2393.

BonazzA, R. 1992 X-ray measurements of shock-induced mixing at an air/xenon interface. $\mathrm{PhD}$ thesis, California Institute of Technology.

Brouillette, M. 1989 On the interaction of shock waves with contact surfaces between gases of different densities. PhD thesis, California Institute of Technology.

Brouillette, M. \& Sturtevant, B. 1989 Growth induced by multiple shock waves normally incident on plane gaseous interfaces. Physica D 37, 248-263.

Brouillette, M. \& Sturtevant, B. 1993 Experiments on the Richtmyer-Meshkov instability: small-scale perturbations on a plane interface. Phys. Fluids A 5, 916-930.

Chandrasekhar, S. 1961 Hydrodynamic and Hydromagnetic Stability. Oxford University Press.

CoLE, R. H. 1948 Underwater Explosions. Princeton University Press. 
DUfF, R. E., Harlow, F. H. \& HiRT, C. W. 1962 Effects of diffusion on interface instability between gases. Phys. Fluids 5, 417-425.

EMERY, M. H., Gardner, J. H. \& Boris, J. 1982 Rayleigh-Taylor and Kelvin-Helmoltz instability in targets accelerated by laser ablation. Phys. Rev. Lett. 48, 677-680.

Frost, D. L. 1988 Dynamics of explosive boiling of a droplet. Phys. Fluids 31, $2554-2561$.

Grun, J., Emery, M. H., Kacenjar, S., Opal, C. B., Mclean, E. A., Obenschain, S., Ripin, B. H. \& Sснмтт, A. 1984 Observation of the Rayleigh-Taylor instability in ablatively accelerated foils. Phys. Rev. Lett. 53, 1352-1355.

KIANG, R. L. 1969 Nonlinear Theory of inviscid Taylor instability near the cutoff wavenumber. Phys. Fluids 12, 1333-1339.

KLINE, S. J. 1967 Observed structure features in turbulent and transitional boundary layers. In Fluid Mechanics of Internal Flow, pp. 27-68. Elsevier.

LeLevier, R., Lasher, G. J. \& BJorklund, F. 1955 Effect of a density gradient on Taylor instability. Rep. UCRL-4459. University of California Radiation Laboratory.

LiNDL, J. D. \& MEAD, W. C. 1975 Two-dimensional simulation of fluid instability in laser-fusion pellet. Phys. Rev. Lett. 34, 1273-1276.

Marble, F. E., Hendricks, G. J. \& Zukoski, E. E. 1987 Progress toward shock enhancement of supersonic combustion processes. AIAA Paper 87-1880.

Markstein, G. H. 1957 a A shock tube study of flame front-pressure wave interaction. In Sixth Symp. (Intl) on Combustion, pp. 387-390. Reinhold.

MARKStein, G. H. $1957 b$ Flow disturbances induced near a slightly wavy contact surface, or flame front, traversed by a shock wave. J. Aero. Sci. 24, 238-239.

Meshkov, E. E. 1969 Instability of the interface of two gases accelerated by a shock wave. Sov. Fluid Dyn. 4, 101-108.

Meyer, K. A. \& Blewett, P. J. 1972 Numerical investigation of the stability of a shock accelerated interface between two fluids. Phys. Fluids 15, 753-757.

Mikaelian, K. O. 1985 Richtmyer-Meshkov instabilities in stratified fluids. Phys. Rev. A 31, 410-419.

Plesset, M. S. 1954 On the stability of fluid flows with spherical symmetry. J. Appl. Phys. 25, 96-98.

RAYLEIGH, LORD 1900 Investigation of the character of the equilibrium of an incompressible heavy fluid of variable density. In Scientific Papers, Vol. 2, p. 200. Dover.

READ, K. I. 1984 Experimental investigation of turbulent mixing by Rayleigh-Taylor instability. Physica D 12, 45-58.

RichTMYER, R. D. 1960 Taylor instability in shock acceleration of compressible fluids. Commun. Pure Appl. Maths 8, 297-319.

SaffMan, P. G. \& MeIron, D. 1989 Kinetic energy generated by the incompressible Richtmyer-Meshkov instability in a continuously stratified fluid. Phys. Fluids A 1, 1767-1771.

Sharp, D. H. 1984 An overview of Rayleigh-Taylor instability. Physica D 12, 3-18.

Smarr, L., Wilson, J. R., Barton, R. T. \& Bowers, R. L. 1981 Rayleigh-Taylor overturn in supernova core collapse. Astrophys. J. 246, 515-525.

Stalker, R. J. \& Crane, K. C. A. 1978 Driver gas contamination in a high-enthalpy reflected shock tunnel. AIAA J. 16, 277-279.

StURTEVAnT, B. 1988 Rayleigh-Taylor instability in compressible fluids. In Shock Tubes and Waves (ed. H. Grönig), pp. 89-100. Wendheim: VCH.

TAYLOR, G. I. 1950 The instability of liquid surfaces when accelerated in a direction perpendicular to their planes, I. Proc. R. Soc. Lond. A 201, 192-196.

Waitz, I. A., Marble, F. E. \& ZuKoski, E. E. 1991 An investigation of a contoured wall injector for hypervelocity mixing augmentation. AIAA Paper 91-2265. 